\title{
Optimality and duality for nonsmooth multiobjective optimization problems
}

Kwan Deok Bae and Do Sang Kim*

${ }^{\text {"Correspondence: }}$ dskim@pknu.ac.kr

Department of Applied

Mathematics, Pukyong National

University, Busan, 608-737, Korea

\section{Springer}

\begin{abstract}
In this paper, we consider a nonsmooth multiobjective programming problems including support functions with inequality and equality constraints. Necessary and sufficient optimality conditions are obtained by using higher-order strong convexity for Lipschitz functions. Mond-Weir type dual problem and duality theorems for a strict minimizer of order $m$ are given.
\end{abstract}

Keywords: nonsmooth multiobjective programming; strict minimizers; optimality conditions; duality

\section{Introduction}

Nonlinear analysis problems are a new and vital area of optimization theory, mathematical physics, economics, engineering and functional analysis. Moreover, nonsmooth problems occur naturally and frequently in optimization.

In 1970, Rockafellar wrote in his book that practical applications are not necessarily differentiable in applied mathematics (see [1]). So, dealing with nondifferentiable mathematical programming problems was very important. Vial [2] studied strongly and weakly convex sets and $\rho$-convex functions.

Auslender [3] introduced the notion of lower second-order directional derivative and obtained necessary and sufficient conditions for a strict local minimizer. Based on Auslender's results, Studniarski [4] proved necessary and sufficient conditions for the problem of the feasible set defined by an arbitrary set. Moreover, Ward [5] derived necessary and sufficient conditions for strict minimizer of order $m$ in nondifferentiable scalar programs. Jimenez [6] introduced the notion of super-strict efficiency for vector problems and gave necessary conditions for strict minimality. Jimenez and Novo [7, 8] obtained first- and second-order optimality conditions for vector optimization problems. Bhatia [9] gave the higher-order strong convexity for Lipschitz functions and established optimality conditions for the new concept of strict minimizer of higher order for a multiobjective optimization problem.

Kim and Bae [10] formulated nondifferentiable multiobjective programs with the support functions. Also, Bae et al. [11] established duality theorems for nondifferentiable multiobjective programming problems under generalized convexity assumptions. Also, Kim and Lee [12] introduced the nonsmooth multiobjective programming problems involving locally Lipschitz functions and support functions. They introduced Karush-Kuhn-Tucker type optimality conditions and established duality theorems for (weak) Pareto-optimal so-

○2013 Bae and Kim; licensee Springer. This is an Open Access article distributed under the terms of the Creative Commons Attribution License (http://creativecommons.org/licenses/by/2.0), which permits unrestricted use, distribution, and reproduction in any medium, provided the original work is properly cited. 
lutions. Recently, Bae and Kim [10] established optimality conditions and duality theorems for a nondifferentiable multiobjective programming problem with support functions.

In this paper, we consider nonsmooth multiobjective programming with inequality and equality constraints. In Section 2, we introduce the concept of a strict minimizer of order $m$ and higher-order strong convexity for this problem. In Section 3, necessary and sufficient optimality theorems are established for a strict minimizer of order $m$ under generalized strong convexity assumptions. In Section 4, we formulate a Mond-Weir type dual problem and obtain weak and strong duality theorems.

\section{Preliminaries}

Let $x, y \in \mathbb{R}^{n}$. The following notation will be used for vectors in $\mathbb{R}^{n}$ :

$$
\begin{aligned}
& x<y \quad \Longleftrightarrow \quad x_{i}<y_{i}, \quad i=1,2, \ldots, n ; \\
& x \leqq y \quad \Longleftrightarrow \quad x_{i} \leqq y_{i}, \quad i=1,2, \ldots, n ; \\
& x \leq y \quad \Longleftrightarrow \quad x_{i} \leqq y_{i}, \quad i=1,2, \ldots, n \text { but } x \neq y ; \\
& x \nless y \text { is the negation of } x<y ; \\
& x \not \leq y \text { is the negation of } x \leq y .
\end{aligned}
$$

For $x, u \in \mathbb{R}, x \leqq u$ and $x<u$ have the usual meaning. Let $\mathbb{R}^{n}$ be the $n$-dimensional Euclidean space, and let $\mathbb{R}_{+}^{n}$ be its nonnegative orthant.

Definition 2.1 [13] Let $D$ be a compact convex set in $\mathbb{R}^{n}$. The support function $s(\cdot \mid D)$ is defined by

$$
s(x \mid D):=\max \left\{x^{T} y: y \in D\right\} .
$$

The support function $s(\cdot \mid D)$ has a subdifferential. The subdifferential of $s(\cdot \mid D)$ at $x$ is given by

$$
\partial s(x \mid D):=\left\{z \in D: z^{T} x=s(x \mid D)\right\} .
$$

The support function $s(\cdot \mid D)$ is convex and everywhere finite, that is, there exists $z \in D$ such that

$$
s(y \mid D) \geq s(x \mid D)+z^{T}(y-x) \quad \text { for all } y \in D .
$$

Equivalently,

$$
z^{T} x=s(x \mid D)
$$

We consider the following multiobjective programming problem.

$\left(\right.$ MOP) Minimize $f(x)+s(x \mid D)=\left(f_{1}(x)+s\left(x \mid D_{1}\right), \ldots, f_{p}(x)+s\left(x \mid D_{p}\right)\right)$ subject to $g(x) \leqq 0, \quad h(x)=0, \quad x \in X$, 
where $f: X \rightarrow \mathbb{R}^{p}, g: X \rightarrow \mathbb{R}^{q}$ and $h: X \rightarrow \mathbb{R}^{r}$ are locally Lipschitz functions, respectively, and $X$ is the convex set of $\mathbb{R}^{n}$. For each $i \in P=\{1,2, \ldots, p\}, D_{i}$ is a compact convex subset of $\mathbb{R}^{n}$.

Further, let $S:=\left\{x \in X \mid g_{j}(x) \leqq 0, j=1, \ldots, q, h_{l}(x)=0, l=1, \ldots, r\right\}$ be the feasible set of (MOP), $B\left(x^{0}, \epsilon\right)=\left\{x \in \mathbb{R}^{n} \mid\left\|x-x^{0}\right\|<\epsilon\right\}$ be an open ball with center $x^{0}$ and radius $\epsilon$ and $I\left(x^{0}\right):=\left\{j \in\{1, \ldots, q\} \mid g_{j}\left(x^{0}\right)=0\right\}$ be the index set of active constraints at $x^{0}$.

We introduce the following definitions due to Jimenez [6].

Definition 2.2 A point $x^{0} \in X$ is called a strict local minimizer for (MOP) if there exists $\epsilon>0$ such that

$$
f(x)+s(x \mid D) \nless f\left(x^{0}\right)+s\left(x^{0} \mid D\right), \quad \forall x \in B\left(x^{0}, \epsilon\right) \cap X .
$$

Definition 2.3 Let $m \geqq 1$ be an integer. A point $x^{0} \in X$ is called a strict local minimizer of order $m$ for (MOP) if there exist $\epsilon>0$ and $c \in \operatorname{int} \mathbb{R}_{+}^{p}$ such that

$$
f(x)+s(x \mid D) \nless f\left(x^{0}\right)+s\left(x^{0} \mid D\right)+\left\|x-x^{0}\right\|^{m} c, \quad \forall x \in B\left(x^{0}, \epsilon\right) \cap X .
$$

Definition 2.4 Let $m \geqq 1$ be an integer. A point $x^{0} \in X$ is called a strict minimizer of order $m$ for (MOP) if there exists $c \in \operatorname{int} \mathbb{R}_{+}^{p}$ such that

$$
f(x)+s(x \mid D) \nless f\left(x^{0}\right)+s\left(x^{0} \mid D\right)+\left\|x-x^{0}\right\|^{m} c, \quad \forall x \in X .
$$

Definition 2.5 [14] Suppose that $f: X \rightarrow \mathbb{R}$ is Lipschitz on X. Clarke's generalized directional derivative of $f$ at $x \in X$ in the direction $d \in \mathbb{R}^{n}$, denoted by $f^{0}(x, d)$, is defined as

$$
f^{0}(x, d)=\limsup _{y \rightarrow x} \frac{f(y+t d)-f(y)}{t} .
$$

Definition 2.6 [14] Clarke's generalized gradient of $f$ at $x \in X$, denoted by $\partial f(x)$, is defined as

$$
\partial f(x)=\left\{\xi \in \mathbb{R}^{n}: f^{0}(x, d) \geq\langle\xi, d\rangle \forall d \in \mathbb{R}^{n}\right\} .
$$

Definition 2.7 For a nonempty subset $X$ of $\mathbb{R}^{n}$, we denote $X^{*}$, the dual cone of $X$, defined by

$$
X^{*}=\left\{u \in \mathbb{R}^{n} \mid u^{T} x \geqq 0, \forall x \in X\right\} .
$$

Further, for $x^{0} \in X, N_{X}\left(x^{0}\right)$ denotes the normal cone to $X$ at $x^{0}$ defined by

$$
N_{X}\left(x^{0}\right)=\left\{d \in \mathbb{R}^{n} \mid\left\langle d, x-x^{0}\right\rangle \leqq 0, \forall x \in X\right\} .
$$

It is clear that $\left(X-x^{0}\right)^{*}=-N_{X}\left(x^{0}\right)$.

We recall the notion of strong convexity of order $m$ introduced by Lin and Fukushima in [15]. 
Definition 2.8 A function $f: X \rightarrow \mathbb{R}$ is said to be strongly convex of order $m$ on a convex set $X$ if there exists $c>0$ such that for $x_{1}, x_{2} \in X$ and $t \in[0,1]$,

$$
f\left(t x_{1}+(1-t) x_{2}\right) \leqq t f\left(x_{1}\right)+(1-t) f\left(x_{2}\right)-c t(1-t)\left\|x_{1}-x_{2}\right\|^{m}
$$

Proposition 2.1 [15] If $f_{i}, i=1, \ldots, p$, are strongly convex of order $m$ on a convex set $X$, then $\sum_{i=1}^{p} t_{i} f_{i}$ and $\max _{1 \leq i \leq p} f_{i}$ are also strongly convex of order $m$ on $X$, where $t_{i} \geq 0, i=1, \ldots, p$.

Definition 2.9 A locally Lipschitz function $f$ is said to be strongly quasiconvex of order $m$ on $\mathrm{X}$ if there exists a constant $c>0$ such that for $x_{1}, x_{2} \in X$,

$$
f\left(x_{1}\right) \leqq f\left(x_{2}\right) \quad \Longrightarrow \quad\left\langle\xi, x_{1}-x_{2}\right\rangle+\left\|x_{1}-x_{2}\right\|^{m} c \leqq 0, \quad \forall \xi \in \partial f\left(x_{2}\right) .
$$

For each $k \in\{1, \ldots, p\}$ and $x \in X$, we consider the following scalarizing problem of (MOP) due to the one in [16].

$$
\begin{array}{lll}
\left(P_{k}\left(x^{0}\right)\right) \quad \text { Minimize } & f_{k}(x)+s\left(x \mid D_{k}\right) \\
\text { subject to } & f_{i}(x)+s\left(x \mid D_{i}\right) \leqq f_{i}\left(x^{0}\right)+s\left(x^{0} \mid D_{i}\right), \quad k \in P, i \neq k, \\
& g_{j}(x) \leqq 0, \quad j=1, \ldots, q, \quad h_{l}(x)=0, \quad l=1, \ldots, r .
\end{array}
$$

The following definition is due to the one in [17].

Definition 2.10 Let $x^{0}$ be a feasible solution for (MOP). We say that the basic regularity condition (BRC) is satisfied at $x^{0}$ if there exist no non-zero scalars $\lambda_{i}^{0} \geqq 0, w_{i} \in D_{i}, i=$ $1, \ldots, p, i \neq k, k \in P, \mu_{j}^{0} \geqq 0, j \in I\left(x^{0}\right), \mu_{j}^{0}=0, j \notin I\left(x^{0}\right)$, and $v_{l}^{0}, l=1, \ldots, r$, such that

$$
0 \in \sum_{i=1, i \neq k}^{p} \lambda_{i}^{0}\left(\partial f_{i}\left(x^{0}\right)+w_{i}\right)+\sum_{j=1}^{q} \mu_{j}^{0} \partial g_{j}\left(x_{0}\right)+\sum_{l=1}^{r} v_{l}^{0} \partial h_{l}\left(x^{0}\right)+N_{X}\left(x^{0}\right) .
$$

\section{Optimality conditions}

In this section, we establish Fritz John necessary optimality conditions, Karush-KuhnTucker necessary optimality conditions and Karush-Kuhn-Tucker sufficient optimality condition for a strict minimizer of (MOP).

Theorem 3.1 (Fritz John necessary optimality conditions) Suppose that $x^{0}$ is a strict minimizer of order $m$ for (MOP) and $f_{i}, i=1, \ldots, p, g_{j}, j=1, \ldots, q$, and $h_{l}, l=1, \ldots, r$, are locally Lipschitz functions at $x^{0}$. Then there exist $\lambda_{i}^{0} \geqq 0, w_{i}^{0} \in D_{i}, i=1, \ldots, p, \mu_{j}^{0} \geqq 0, j=1, \ldots, q$, and $v_{l}^{0}, l=1, \ldots, r$, not all zero such that

$$
\begin{aligned}
& 0 \in \sum_{i=1}^{p} \lambda_{i}^{0}\left(\partial f_{i}\left(x^{0}\right)+w_{i}^{0}\right)+\sum_{j=1}^{q} \mu_{j}^{0} \partial g_{j}\left(x^{0}\right)+\sum_{l=1}^{r} \nu_{l}^{0} \partial h_{l}\left(x^{0}\right)+N_{X}\left(x^{0}\right), \\
& \left\langle w_{i}^{0}, x^{0}\right\rangle=s\left(x^{0} \mid D_{i}\right), \quad i=1, \ldots, p, \\
& \mu_{j}^{0} g_{j}\left(x^{0}\right)=0, \quad j=1, \ldots, q .
\end{aligned}
$$


Proof Since $x^{0}$ is a strict minimizer of order $m$ for (MOP), it is a strict minimizer for (MOP). It can be shown that $x^{0}$ solves the following problem:

$$
\begin{array}{ll}
\text { minimize } & F(x) \\
\text { subject to } & g(x) \leqq 0,
\end{array}
$$

where

$$
\begin{aligned}
F(x)= & \max \left\{\left(f_{1}(x)+s\left(x \mid D_{1}\right)\right)-\left(f_{1}\left(x^{0}\right)+s\left(x^{0} \mid D_{1}\right)\right), \ldots,\right. \\
& \left.\left(f_{p}(x)+s\left(x \mid D_{p}\right)\right)-\left(f_{p}\left(x^{0}\right)+s\left(x^{0} \mid D_{p}\right)\right)\right\} .
\end{aligned}
$$

If it is not so, then there exits $x^{1} \in \mathbb{R}^{n}$ such that $F\left(x^{1}\right)<F\left(x^{0}\right), g\left(x^{1}\right) \leqq 0, h\left(x^{1}\right)=0$. Since $F\left(x^{0}\right)=0$, we have $F\left(x^{1}\right)<0$. This contradicts the fact that $x^{0}$ is a strict minimizer for (MOP). Since $x^{0}$ minimizes $F(x)$, from Theorem 6.1.1 in Clarke [14], there exists $(\lambda, \mu, v) \in$ $\left(\mathbb{R}^{p}, \mathbb{R}^{q}, \mathbb{R}^{r}\right)$ not all zero such that

$$
0 \in \sum_{i=1}^{p} \lambda_{i} \partial F\left(x^{0}\right)+\sum_{j \in I\left(x^{0}\right)} \mu_{j} \partial g_{j}\left(x^{0}\right)+\sum_{l=1}^{r} \nu_{l} \partial h_{l}\left(x^{0}\right)+N_{X}\left(x^{0}\right)
$$

Letting $\mu_{j}=0$, for $j \notin I\left(x^{0}\right)$, we have

$$
0 \in \sum_{i=1}^{p} \lambda_{i} \partial F\left(x^{0}\right)+\sum_{j=1}^{q} \mu_{j} \partial g_{j}\left(x^{0}\right)+\sum_{l=1}^{r} \nu_{l} \partial h_{l}\left(x^{0}\right)+N_{X}\left(x^{0}\right) .
$$

Since $F(x)=\max \left\{(f(x)+s(x \mid D))-\left(f\left(x^{0}\right)+s\left(x^{0} \mid D\right)\right)\right\}$ for any $x \in X$ and $s\left(x^{0} \mid D_{i}\right)=\left(x^{0}\right)^{T} w_{i}$, $i=1, \ldots, p$, we have

$$
\begin{aligned}
\partial F\left(x^{0}\right) & \subset \operatorname{co}\left\{\partial\left(f_{i}\left(x^{0}\right)+s\left(x^{0} \mid D_{i}\right)\right)\right\} \\
& =\operatorname{co}\left\{\left(\partial f_{i}\left(x^{0}\right)+w_{i}\right)\right\}
\end{aligned}
$$

where $\operatorname{co}\left\{\partial\left(f_{i}\left(x^{0}\right)+s\left(x^{0} \mid D_{i}\right)\right)\right\}$ denotes the convex hull of $\left\{\partial\left(f_{i}\left(x^{0}\right)+s\left(x^{0} \mid D_{i}\right)\right)\right\}$. Hence, there exist $\lambda_{i}^{0} \geqq 0, w_{i}^{0} \in D_{i}, i=1, \ldots, p, \mu_{j}^{0} \geqq 0, j=1, \ldots, q$, and $v_{l}, l=1, \ldots, r$, not all zero such that

$$
\begin{aligned}
& 0 \in \sum_{i=1}^{p} \lambda_{i}\left(\partial f_{i}\left(x^{0}\right)+w_{i}^{0}\right)+\sum_{j=1}^{q} \mu_{j} \partial g_{j}\left(x^{0}\right)+\sum_{l=1}^{r} \nu_{l} \partial h_{l}\left(x^{0}\right)+N_{X}\left(x^{0}\right), \\
& \left\langle w_{i}^{0}, x^{0}\right\rangle=s\left(x^{0} \mid D_{i}\right), \quad i=1, \ldots, p, \\
& \mu_{j}^{0} g_{j}\left(x^{0}\right)=0, \quad j=1, \ldots, q .
\end{aligned}
$$

Theorem 3.2 (Karush-Kuhn-Tucker necessary optimality conditions) Suppose that $x^{0}$ is a strict minimizer of order $m$ for (MOP) and $f_{i}, i=1, \ldots, p, g_{j}, j=1, \ldots, q$, and $h_{l}, l=1, \ldots, r$, are locally Lipschitz functions at $x^{0}$. If the basic regularity condition $(B R C)$ holds at $x^{0}$, then there exist $\lambda_{i}^{0} \geqq 0, w_{i}^{0} \in D_{i}, i=1, \ldots, p, \mu_{j}^{0} \geqq 0, j=1, \ldots, q$, and $v_{l}^{0}, l=1, \ldots, r$, such that 


$$
\begin{aligned}
& 0 \in \sum_{i=1}^{p} \lambda_{i}^{0}\left(\partial f_{i}\left(x^{0}\right)+w_{i}^{0}\right)+\sum_{j=1}^{q} \mu_{j}^{0} \partial g_{j}\left(x^{0}\right)+\sum_{l=1}^{r} \nu_{l}^{0} \partial h_{l}\left(x^{0}\right)+N_{X}\left(x^{0}\right), \\
& \left\langle w_{i}^{0}, x^{0}\right\rangle=s\left(x^{0} \mid D_{i}\right), \quad i=1, \ldots, p, \\
& \mu_{j}^{0} g_{j}\left(x^{0}\right)=0, \quad j=1, \ldots, q, \\
& \left(\lambda_{1}^{0}, \ldots, \lambda_{p}^{0}\right) \neq(0, \ldots, 0) .
\end{aligned}
$$

Proof Since $x^{0}$ is a strict minimizer of order $m$ for (MOP), by Theorem 3.1, there exist $w_{i}^{0} \in D_{i}, \lambda_{i}^{0} \geqq 0, i=1, \ldots, p, \mu_{j}^{0} \geqq 0, j=1, \ldots, q$, and $v_{l}, l=1, \ldots, r$, not all zero such that

$$
\begin{aligned}
& 0 \in \sum_{i=1}^{p} \lambda_{i}^{0}\left(\partial f_{i}\left(x^{0}\right)+w_{i}^{0}\right)+\sum_{j=1}^{q} \mu_{j}^{0} \partial g_{j}\left(x^{0}\right)+\sum_{l=1}^{r} v_{l}^{0} \partial h_{l}\left(x^{0}\right)+N_{X}\left(x^{0}\right), \\
& \left\langle w_{i}^{0}, x^{0}\right\rangle=s\left(x^{0} \mid D_{i}\right), \quad i=1, \ldots, p, \\
& \mu_{j}^{0} g_{j}\left(x^{0}\right)=0, \quad j=1, \ldots, q .
\end{aligned}
$$

It can be shown that $\left(\lambda_{1}^{0}, \ldots, \lambda_{p}^{0}\right) \neq(0, \ldots, 0)$. If $\lambda_{i}^{0}=0, i=1, \ldots, p$, then we have

$$
0 \in \sum_{i=1, i \neq k}^{p} \lambda_{i}^{0}\left(\partial f_{i}\left(x^{0}\right)+w_{i}\right)+\sum_{j=1}^{q} \mu_{j}^{0} \partial g_{j}\left(x_{0}\right)+\sum_{l=1}^{r} \nu_{l}^{0} \partial h_{l}\left(x^{0}\right)+N_{X}\left(x^{0}\right)
$$

for each $k \in P=\{1, \ldots, p\}$. Since the basic regularity condition (BRC) holds at $x^{0}$, we have $\lambda_{k}=0, k \in P, k \neq i=\{1, \ldots, p\}, \mu_{j}=0, j \in I\left(x^{0}\right)$, and $v_{l}=0, l=1, \ldots, r$. This contradicts the fact that $\lambda_{i}, \lambda_{k}, k \in P, k \neq i, \mu_{j}, j \in I\left(x^{0}\right)$ and $v_{l}, l=1, \ldots, r$, are not all simultaneously zero. Hence, $\left(\lambda_{1}, \ldots, \lambda_{p}\right) \neq(0, \ldots, 0)$.

Theorem 3.3 (Karush-Kuhn-Tucker sufficient optimality conditions) Assume that there exist $\lambda_{i}^{0} \geqq 0, w_{i}^{0} \in D_{i}, i=1, \ldots, p, \mu_{j}^{0} \geqq 0, j=1, \ldots, q$, and $v_{l}^{0}, l=1, \ldots, r$, such that for $x^{0} \in X$,

$$
\begin{aligned}
& 0 \in \sum_{i=1}^{p} \lambda_{i}^{0}\left(\partial f_{i}\left(x^{0}\right)+w_{i}^{0}\right)+\sum_{j=1}^{q} \mu_{j}^{0} \partial g_{j}\left(x^{0}\right)+\sum_{l=1}^{r} v_{l}^{0} \partial h_{l}\left(x^{0}\right)+N_{X}\left(x^{0}\right), \\
& \left\langle w_{i}^{0}, x^{0}\right\rangle=s\left(x^{0} \mid D_{i}\right), \quad i=1, \ldots, p, \\
& \mu_{j}^{0} g_{j}\left(x^{0}\right)=0, \quad j=1, \ldots, q, \\
& \left(\lambda_{1}^{0}, \ldots, \lambda_{p}^{0}\right) \neq(0, \ldots, 0) .
\end{aligned}
$$

Assume further that $f_{i}, i=1, \ldots, p$, are strongly convex of order $m$ on $X, g_{j}, j \in I\left(x^{0}\right)$ are strongly quasiconvex of order $m$ on $X$ and $v^{T} h$ is strongly quasiconvex of order $m$ on $X$. Then $x^{0}$ is a strict minimizer of order $m$ for (MOP).

Proof Since $f_{i}, i=1, \ldots, p$, are strongly convex of order $m$ on $X$ and $(\cdot)^{T} w_{i}, i=1, \ldots, p$, are convex, there exists $c_{i}>0, i=1, \ldots, p$, such that for all $x \in X, \xi_{i} \in \partial f_{i}\left(x^{0}\right)$ and $w_{i} \in D_{i}$, $i=1, \ldots, p$,

$$
f_{i}(x)-f_{i}\left(x^{0}\right) \geqq\left\langle\xi_{i}, x-x^{0}\right\rangle+\left\|x-x^{0}\right\|^{m} c_{i}, \quad x^{T} w_{i}-\left(x^{0}\right)^{T} w_{i} \geqq\left\langle x_{i}, x-x^{0}\right\rangle .
$$


So, we obtain

$$
\left(f_{i}(x)+x^{T} w_{i}\right)-\left(f_{i}\left(x^{0}\right)+\left(x^{0}\right)^{T} x_{i}\right) \geqq\left\langle\xi_{i}+w_{i}, x-x^{0}\right\rangle+\left\|x-x^{0}\right\|^{m} c_{i} .
$$

For $\lambda_{i}^{0} \geqq 0, i=1, \ldots, p,(3.1)$ implies

$$
\begin{gathered}
\sum_{i=1}^{p} \lambda_{i}^{0}\left(f_{i}(x)+x^{T} w_{i}\right)-\sum_{i=1}^{p} \lambda_{i}^{0}\left(f_{i}\left(x^{0}\right)+\left(x^{0}\right)^{T} w_{i}\right) \\
\geqq \sum_{i=1}^{p} \lambda_{i}^{0}\left\langle\xi_{i}+w_{i}, x-x^{0}\right\rangle+\sum_{i=1}^{p} \lambda_{i}^{0}\left\|x-x^{0}\right\|^{m} c_{i} .
\end{gathered}
$$

For $x \in X$, we have

$$
\begin{aligned}
& g_{j}(x) \leqq g_{j}\left(x^{0}\right), \quad j \in I\left(x^{0}\right), \\
& v^{T} h(x)=v^{T} h\left(x^{0}\right) .
\end{aligned}
$$

Since $g_{j}, j \in I\left(x^{0}\right)$ are strongly quasiconvex of order $m$ on $X$ and $v^{T} h$ is strongly quasiconvex of order $m$ on $X$, it follows that there exist $c_{j}>0, \eta_{j} \in \partial g_{j}\left(x^{0}\right), j \in I\left(x^{0}\right), c>0$, and $\zeta \in$ $\partial v^{T} h\left(x^{0}\right)$ such that

$$
\begin{aligned}
& \left\langle\eta_{j}, x-x^{0}\right\rangle+\left\|x-x^{0}\right\|^{m} c_{j} \leqq 0, \\
& \left\langle\zeta, x-x^{0}\right\rangle+\left\|x-x^{0}\right\|^{m} c \leqq 0 .
\end{aligned}
$$

For $\mu_{j}^{0} \geqq 0, j \in I\left(x^{0}\right)$, we obtain

$$
\left\langle\sum_{j \in I\left(x^{0}\right)} \mu_{j}^{0} \eta_{j}, x-x^{0}\right\rangle+\sum_{j \in I\left(x^{0}\right)} \mu_{j}^{0}\left\|x-x^{0}\right\|^{m} c_{j} \leqq 0 .
$$

Since $\mu_{j}^{0}=0$ for $j \notin I\left(x^{0}\right)$, (3.4) implies

$$
\left\langle\sum_{j=1}^{q} \mu_{j}^{0} \eta_{j}, x-x^{0}\right\rangle+\sum_{j=1}^{q} \mu_{j}^{0}\left\|x-x^{0}\right\|^{m} c_{j} \leqq 0 .
$$

By (3.2), (3.3) and (3.5), we get

$$
\sum_{i=1}^{p} \lambda_{i}^{0}\left(f_{i}(x)+x^{T} w_{i}\right)-\sum_{i=1}^{p} \lambda_{i}^{0}\left(f_{i}\left(x^{0}\right)+\left(x^{0}\right)^{T} w_{i}\right) \geqq\left\|x-x^{0}\right\|^{m} a,
$$

where $a=\sum_{i=1}^{p} \lambda_{i}^{0} c_{i}+\sum_{j=1}^{q} \mu_{j}^{0} c_{j}+\sum_{l=1}^{m} v_{l}^{0} c_{l}$. This implies that

$$
\sum_{i=1}^{p} \lambda_{i}^{0}\left[\left(f_{i}(x)+x^{T} w_{i}\right)-\left(f_{i}\left(x^{0}\right)+\left(x^{0}\right)^{T} w_{i}\right)-\left\|x-x^{0}\right\|^{m} d_{i}\right] \geqq 0,
$$

where $d=a e$. 
Suppose that $x^{0}$ is not a strict minimizer of order $m$ for (MOP). Then there exist $x, x^{0} \in X$ and $c \in \mathbb{R}_{+}^{p}$ such that

$$
f(x)+s(x \mid D)<f\left(x^{0}\right)+s\left(x^{0} \mid D\right)+\left\|x-x^{0}\right\|^{m} c, \quad \forall x \in X .
$$

Since $x^{T} w \leqq s(x \mid D)$ and $\left(x^{0}\right)^{T} w=s\left(x^{0} \mid D\right)$, we have

$$
\begin{aligned}
f(x)+x^{T} w & \leqq f(x)+s(x \mid D) \\
& <f\left(x^{0}\right)+s\left(x^{0} \mid D\right)+\left\|x-x^{0}\right\|^{m} c \\
& =f\left(x^{0}\right)+\left(x^{0}\right)^{T} w+\left\|x-x^{0}\right\|^{m} c .
\end{aligned}
$$

For $\lambda_{i}^{0} \geqq 0$, we obtain

$$
\sum_{i=1}^{p} \lambda_{i}^{0}\left(f_{i}(x)+x^{T} w_{i}\right)<\sum_{i=1}^{p} \lambda_{i}^{0}\left(f_{i}\left(x^{0}\right)+\left(x^{0}\right)^{T} w_{i}\right)+\sum_{i=1}^{p} \lambda_{i}^{0}\left\|x-x^{0}\right\|^{m} c_{i} .
$$

This is a contradiction to (3.6).

Remark 3.1 Suppose that $g_{j}, j \in I\left(x^{0}\right)$ are strongly convex of order $m$ on $X$ and that $v^{T} h$ is strongly convex of order $m$ on $X$. Then the conclusion of Theorem 3.3 also holds.

Proof It follows on the lines of Theorem 3.3.

\section{Duality theorems}

Now we propose the following Mond-Weir type dual (MOD) to (MOP):

$$
\begin{array}{ll}
\text { (MOD) Maximize } & f(u)+u^{T} w \\
\text { subject to } \quad 0 \in \sum_{i=1}^{p} \lambda_{i}\left(\partial f_{i}(u)+w_{i}\right)+\sum_{j=1}^{q} \mu_{j} \partial g_{j}(u) & \\
& +\sum_{l=1}^{r} \nu_{l} \partial h_{l}(u)+N_{X}(u), \\
& \sum_{j=1}^{q} \mu_{j} g_{j}(u)+\sum_{l=1}^{r} v_{l} h_{l}(u) \geqq 0, \\
& \lambda_{i} \geqq 0, \quad w_{i} \in D_{i}, \quad i=1, \ldots, p, \lambda^{T} e=1, \\
& \mu_{j} \geqq 0, \quad j=1, \ldots, q, \quad v_{l}, \quad l=1, \ldots, r, u \in X .
\end{array}
$$

Theorem 4.1 (Weak duality) Let $x$ and $(u, w, \lambda, \mu, \nu)$ be feasible solutions of (MOP) and (MOD), respectively. If $f_{i}, i=1, \ldots, p$, are strongly convex of order $m$ at $u$ and $\sum_{j=1}^{q} \mu_{j} g_{j}(\cdot)+$ $\sum_{l=1}^{r} v_{l} h_{l}(\cdot)$ is strongly quasiconvex of order $m$ at $u$, then the following cannot hold:

$$
f_{i}(x)+s\left(x \mid D_{i}\right)<f_{i}(u)+u^{T} w_{i}, \quad i=1, \ldots, p .
$$


Proof Since $x$ is a feasible solution of (MOP) and $(u, w, \lambda, \mu, v)$ is a feasible solution of (MOD), we have

$$
\sum_{j=1}^{q} \mu_{j} g_{j}(x)+\sum_{l=1}^{r} \mu_{l} h_{l}(x) \leqq \sum_{j=1}^{q} \mu_{j} g_{j}(u)+\sum_{l=1}^{r} \mu_{l} h_{l}(u)
$$

Since $\sum_{j=1}^{q} \mu_{j} g_{j}(\cdot)+\sum_{l=1}^{r} v_{l} h_{l}(\cdot)$ is strongly quasiconvex of order $m$ at $u$, it follows that there exist $c_{j}>0, \eta_{j} \in \partial g_{j}(u), j=1, \ldots, q, c_{l}>0$, and $\zeta_{l} \in \partial h_{l}(u)$ such that

$$
\left\langle\sum_{j=1}^{q} \mu_{j} \eta_{j}+\sum_{l=1}^{r} v_{l} \zeta_{l}, x-u\right\rangle+\sum_{j=1}^{q}\|x-u\|^{m} c_{j}+\sum_{l=1}^{r}\|x-u\|^{m} c_{l} \leqq 0 .
$$

Now, suppose contrary to the result that (4.5) holds. Since $x^{T} w_{i} \leqq s\left(x \mid D_{i}\right), i=1, \ldots, p$, we obtain

$$
f_{i}(x)+x^{T} w_{i}<f_{i}(u)+u^{T} w_{i}, \quad i=1, \ldots, p
$$

For $c \in$ int $\mathbb{R}_{+}^{p}$, we obtain

$$
f_{i}(x)+x^{T} w_{i}<f_{i}(u)+u^{T} w_{i}+\|x-u\|^{m} c_{i}, \quad i=1, \ldots, p .
$$

For $\lambda_{i} \geqq 0$, we obtain

$$
\sum_{i=1}^{p} \lambda_{i}\left(f_{i}(x)+x^{T} w_{i}\right)<\sum_{i=1}^{p} \lambda_{i}\left(f_{i}(u)+u^{T} w_{i}\right)+\sum_{i=1}^{p} \lambda_{i}\|x-u\|^{m} c_{i} .
$$

Since $f_{i}, i=1, \ldots, p$, are strongly convex of order $m$ at $u$ and $(\cdot)^{T} w_{i}, i=1, \ldots, p$, are convex at $u$, there exists $c_{i}>0, i=1, \ldots, p$, such that for all $x \in X, \xi_{i} \in \partial f_{i}\left(x^{0}\right)$ and $w_{i} \in D_{i}, i=1, \ldots, p$,

$$
\begin{aligned}
& f_{i}(x)-f_{i}(u) \geqq\left\langle\xi_{i}, x-u\right\rangle+\|x-u\|^{m} c_{i}, \\
& x^{T} w_{i}-u^{T} w_{i} \geqq\left\langle w_{i}, x-u\right\rangle .
\end{aligned}
$$

So, we obtain

$$
\left(f_{i}(x)+x^{T} w_{i}\right)-\left(f_{i}(u)+u^{T} w_{i}\right) \geqq\left\langle\xi_{i}+w_{i}, x-u\right\rangle+\|x-u\|^{m} c_{i}
$$

For $\lambda_{i} \geqq 0, i=1, \ldots, p$, we obtain

$$
\begin{aligned}
& \sum_{i=1}^{p} \lambda_{i}\left(f_{i}(x)+x^{T} w_{i}\right)-\sum_{i=1}^{p} \lambda_{i}\left(f_{i}(u)+u^{T} w_{i}\right) \\
& \quad \geqq\left\langle\sum_{i=1}^{p} \lambda_{i}\left(\xi_{i}+w_{i}\right), x-u\right\rangle+\sum_{i=1}^{p} \lambda_{i}\|x-u\|^{m} c_{i} .
\end{aligned}
$$

By (4.6) and (4.10), we get

$$
\sum_{i=1}^{p} \lambda_{i}\left(f_{i}(x)+x^{T} w_{i}\right)-\sum_{i=1}^{p} \lambda_{i}\left(f_{i}(u)+u^{T} w_{i}\right) \geqq\|x-u\|^{m} a,
$$


where $a=\sum_{i=1}^{p} \lambda_{i} c_{i}+\sum_{j=1}^{q} \mu_{j} c_{j}+\sum_{l=1}^{r} v_{l} c_{l}$. This implies that

$$
\sum_{i=1}^{p} \lambda_{i}\left[\left(f_{i}(x)+x^{T} w_{i}\right)-\left(f_{i}(u)+u^{T} w_{i}\right)-\|x-u\|^{m} d_{i}\right] \geqq 0,
$$

where $d=a e$, since $\lambda^{T} e=1$. This is a contradiction to (4.8).

Lemma 4.1 If $g_{j}(\cdot), j=1, \ldots, m$, are strongly convex of order $m$ on $X$ and $v^{T} h$ is strongly convex of order $m$ on $X$, then the same conclusion of Theorem 4.1 also holds.

Proof It follows on the lines of Theorem 4.1.

Definition 4.1 Let $m \geqq 1$ be an integer. A point $x^{0} \in X$ is called a strict maximizer of order $m$ for (MOD) if there exists $c \in$ int $\mathbb{R}_{+}^{p}$ such that

$$
f\left(x^{0}\right)+\left(x^{0}\right)^{T} w+\left\|x-x^{0}\right\|^{m} c \nless f(x)+x^{T} w, \quad \forall x \in X .
$$

Theorem 4.2 (Strong duality) If $x^{0}$ is a strict minimizer of order $m$ for (MOP) and the basic regularity condition $(B R C)$ holds at $x^{0}$, then there exist $\lambda_{i}^{0} \geqq 0, w_{i}^{0} \in D_{i}, i=1, \ldots, p$, $\mu_{j}^{0} \geqq 0, j=1, \ldots, q$, and $v_{l}^{0}, l=1, \ldots, r$, such that $\left(x^{0}, w^{0}, \lambda^{0}, \mu^{0}, v^{0}\right)$ is a feasible solution of (MOD) and $\left(x^{0}\right)^{T} w_{i}^{0}=s\left(x^{0} \mid D_{i}\right), i=1, \ldots, p$. Moreover, if the assumptions of Theorem 4.1 are satisfied, then $\left(x^{0}, w^{0}, \lambda^{0}, \mu^{0}, \nu^{0}\right)$ is a strict maximizer of order $m$ for (MOD).

Proof By Theorem 3.3, there exists $\lambda_{i}^{0} \geqq 0, w_{i}^{0} \in D_{i}, i=1, \ldots, p, \mu_{j}^{0} \geqq 0, j=1, \ldots, q$, and $\nu_{l}^{0}$, $l=1, \ldots, r$, such that

$$
\begin{aligned}
& 0 \in \sum_{i=1}^{p} \lambda_{i}^{0}\left(\partial f_{i}\left(x^{0}\right)+w_{i}^{0}\right)+\sum_{j=1}^{q} \mu_{j}^{0} \partial g_{j}\left(x^{0}\right)+\sum_{l=1}^{r} \nu_{l}^{0} \partial h_{l}\left(x^{0}\right)+N_{X}\left(x^{0}\right), \\
& \left\langle w_{i}^{0}, x^{0}\right\rangle=s\left(x^{0} \mid D_{i}\right), \quad i=1, \ldots, p, \\
& \mu_{j}^{0} g_{j}\left(x^{0}\right)=0, \quad j=1, \ldots, q, \\
& \left(\lambda_{1}^{0}, \ldots, \lambda_{p}^{0}\right) \neq(0, \ldots, 0) .
\end{aligned}
$$

Thus $\left(x^{0}, w^{0}, \lambda^{0}, \mu^{0}, v^{0}\right)$ is a feasible solution of (MOD) and $\left(x^{0}\right)^{T} w_{i}^{0}=s\left(x^{0} \mid D_{i}\right), i=1, \ldots, p$. By Theorem 4.1, we obtain that the following holds:

$$
\begin{aligned}
f_{i}\left(x^{0}\right)+\left(x^{0}\right)^{T} w_{i}^{0} & =f_{i}\left(x^{0}\right)+s\left(x^{0} \mid D_{i}\right) \\
& \nless f_{i}(u)+u^{T} w_{i}, \quad i=1, \ldots, p,
\end{aligned}
$$

for a given feasible solution $(u, w, \lambda, \mu, v)$ of (MOD). For $x^{0}, u \in X$ and $c \in \operatorname{int} \mathbb{R}^{p}$, we have

$$
\begin{gathered}
f\left(x^{0}\right)+\left(x^{0}\right)^{T} w^{0}+\left\|u-x^{0}\right\|^{m} c \\
\not f(u)+u^{T} w .
\end{gathered}
$$

Thus, $\left(x^{0}, w^{0}, \lambda^{0}, \mu^{0}, v^{0}\right)$ is a strict maximizer of order $m$ for (MOD). 
Remark 4.1 Theorem 4.1 and Theorem 4.2 reduce to [13, Theorem 4.1 and Theorem 4.2] in an inequality constraint case. More exactly, $f_{i}(\cdot)+(\cdot)^{T} w_{i}, i=1, \ldots, p$, and $g_{j}(\cdot), j \in I(u)$ at the considered point in the framework of [13, Theorem 4.1 and Theorem 4.2] are strongly convex of order $m$ and strongly quasiconvex of order $m$, respectively.

\section{Competing interests}

The authors declare that they have no competing interests.

\section{Authors' contributions}

DSK obtained necessary and sufficient optimality conditions by using higher-order strong convexity of Lipschitz functions, formulated a Mond-Weir type dual problem and established weak and strong duality theorems for a strict minimizer of order $m$. KDB carried out the duality studies and participated in the sequence alignment and drafted the manuscript. All authors read and approved the final manuscript.

\section{Acknowledgements}

This research was supported by the Basic Science Research Program through the National Research Foundation of Korea (NRF) funded by the Ministry of Education, Science and Technology (NRF-2013R1A1A2A10008908).

Received: 24 July 2013 Accepted: 21 October 2013 Published: 22 Nov 2013

\section{References}

1. Rockafellar, RT: Convex Analysis. Princeton University Press, Princeton (1970)

2. Vial, JP: Strong and weak convexity of sets and functions. Math. Oper. Res. 8, 231-259 (1983)

3. Auslender, A: Stability in mathematical programming with non-differentiable data. SIAM J. Control Optim. 22, 239-254 (1984)

4. Studniarski, M: Necessary and sufficient conditions for isolated local minima of nonsmooth functions. SIAM J. Control Optim. 24, 1044-1049 (1986)

5. Ward, DE: Characterizations of strict local minima and necessary conditions for weak sharp minima. J. Optim. Theory Appl. 80, 551-571 (1994)

6. Jimenez, B: Strictly efficiency in vector optimization. J. Math. Anal. Appl. 265, 264-284 (2002)

7. Jimenez, B, Novo, V: First and second order sufficient conditions for strict minimality in multiobjective programming. Numer Funct. Anal. Optim. 23, 303-322 (2002)

8. Jimenez, B, Novo, V: First and second order sufficient conditions for strict minimality in nonsmooth vector optimization. J. Math. Anal. Appl. 284, 496-510 (2003)

9. Bhatia, G: Optimality and mixed saddle point criteria in multiobjective optimization. J. Math. Anal. Appl. 342, 135-145 (2008)

10. Kim, DS, Bae, KD: Optimality conditions and duality for a class of nondifferentiable multiobjective programming problems. Taiwan. J. Math. 13(2B), 789-804 (2009)

11. Bae, KD, Kang, YM, Kim, DS: Efficiency and generalized convex duality for nondifferentiable multiobjective programs. J. Inequal. Appl. 2010, Article ID 930457 (2010)

12. Kim, DS, Lee, HJ: Optimality conditions and duality in nonsmooth multiobjective programs. J. Inequal. Appl. 2010, Article ID 939537 (2010)

13. Bae, KD, Kim, DS: Optimality and duality theorems in nonsmooth multiobjective optimization. Fixed Point Theory Appl. 2011, Article ID 42 (2011)

14. Clarke, FH: Optimization and Nonsmooth Analysis. Wiley-Interscience, New York (1983)

15. Lin, GH, Fukushima, M: Some exact penalty results for nonlinear programs and mathematical programs with equilibrium constraints. J. Optim. Theory Appl. 118,67-80 (2003)

16. Chankong, V, Haimes, YY: Multiobjective Decision Making: Theory and Methodology. North-Holland, New York (1983)

17. Chandra, S, Dutta, J, Lalitha, CS: Regularity conditions and optimality in vector optimization. Numer. Funct. Anal. Optim. 25, 479-501 (2004) 